\title{
A Novel Intelligent Cluster-Head (ICH) to Mitigate the Handover Problem of Clustering in VANETs
}

\author{
A.H. Abbas* ${ }^{1}$, Mohammed I. Habelalmateen ${ }^{2}$, L. Audah ${ }^{3}$, N.A.M. Alduais ${ }^{4}$ \\ Wireless and Radio Science Centre (WARAS) Faculty of Electrical and Electronic Engineering \\ Universiti Tun Hussein Onn Malaysia 86400 Parit Raja, Batu Pahat, Johor, Malaysia ${ }^{1,2,3,4}$ \\ Department of Computer Technical Engineering, College of Technical Engineering \\ The Islamic University, 54001 Najaf, Iraq ${ }^{2}$
}

\begin{abstract}
The huge development in the number of Vehicle factories have resulted in many people having lost their life due to accident, which has made vehicular Ad-hoc networks (VANETs) hot topic to enable improved communication between vehicles aimed at reducing the loss of life. The main challenge in this area is vehicle mobility, which has direct effect on network stability. Thus, most previous studies that discussed clustering focused on cluster formation, cluster-head selection and the stability of cluster to reduce the impact of mobility in the network, with little attention given to the clusters when passing from base-station to neighbor base-station. Therefore, this study focused on handover problem that occurs after cluster formation and cluster-head election during cluster passing from base station to base station, known as overlapping area. As the cluster in an overlapping area receives two signals from different base stations, the signal arriving at the cluster becomes weak due to interference between two frequencies resulting in loss of cluster information in the overlapping area. In this study, proposed a novel method named Intelligent Cluster-Head (ICH), which is a controller on two clusters that are used to change uplink between clusters to solve the handover problem in the overlapping area. The proposed method was evaluated with VMaSC-1hop method. The proposed method achieved percentage of packet loss up to $0.8 \%$, percentage of packet delivery ratio (PDR) $99 \%$, percentage of number of disconnected links $0.12 \%$ and percentage of network efficiency $99 \%$ in the cells edge.
\end{abstract}

Keywords-Vehicular Ad-Hoc networks; ITS; clustering; overlapping area; handover; ICH

\section{INTRODUCTION}

This template, Vehicular Ad-hoc Networks (VANETs) is a sub-part of mobile Ad-hoc networks (MANETs). The main idea of VANETs is to establish communication between vehicles for the personal safety of the vehicle's occupants. VANETs has two main types of communication: 1) vehicle to vehicle $(\mathrm{V} 2 \mathrm{~V})$, which allows vehicles to communicate between others in point to point link by using IEEE802.11p standard protocol. The advantages of this type are free of cost, no infrastructure required and easy network deployment. However, it triggers some issues when there are an insufficient number of vehicles, which result in disconnect problem and packet loss [1], [2]; 2) vehicle to infrastructure (V2I), which solves this issue by making sure that the vehicles are directly connected to the base station (BS). BS coverage is large because the higher transmission range decreases disconnect problem [3], [4]. However, there are still some issues that arise in this type, such as cost, difficult deployment network and network load. All of the above issues result from high vehicle mobility. High speed of vehicles causes a change in topology, which results in an unstable network. Therefore, clustering is used to reduce the above issues by combining vehicles in a group called cluster. Cluster means connecting a number of vehicles that are in the same transmission range. One of these vehicles is called cluster-head $(\mathrm{CH})$ and the remaining vehicles are called cluster-members (CMs), with the $\mathrm{CH}$ responsible for managing intra and inter-cluster communication [5]. The benefit of clustering enhancement network performance is that it reduces connection congestion at the base-stations in the network. Nevertheless, vehicle mobility is still the main challenge in the clustering according to [6]-[9]. Most of the previous studies focused on reducing the effect of mobility by increasing cluster stability since VANETs is sub-part of MANETs. However, although the maximum nodes speed are 8 $\mathrm{km} / \mathrm{h}$ [10], the handover between neighboring base-stations (BS) in MANETs is a big challenge. Therefore, handover has become a huge challenge in VANETs. According to the National Speed Limits of Malaysia, the speed limit in urban areas is $90 \mathrm{~km} / \mathrm{h}$. In addition, according to local cellular networks in Malaysia, the average coverage area of LTE-BS in the city is between 300 and 400 meters, which leads to vehicles passing from one BS to another in short time duration, resulting in more vehicle information required during handover. Therefore, this paper has proposed a new method to solve the handover in the overlapping area, a method called Intelligent Cluster-Head (ICH). This paper is organized as follows; section II is focused on handover problem, section III described previous work related to a heterogeneous network, section IV describes the new method (ICH) and theoretical analysis, section $\mathrm{V}$ presents simulation and result analysis, section VI presents the conclusion and future work.

The introduction should briefly place the study in a broad context and highlight why it is important. It should define the purpose of the work and its significance. The current state of the research field should be reviewed carefully, and key publications cited. Please highlight controversial and diverging hypotheses when necessary. Finally, briefly mention the main aim of the work and highlight the principal conclusions. As far as possible, please keep the introduction comprehensible to scientists outside your particular field of research. References should be numbered in order of appearance and indicated by a numeral or numerals in square brackets, e.g., [1] or [2,3], or [4-6]. See the end of the document for further details on references. 


\section{HANDOVER PRoblem StATEMENT}

This section discussed the handover problems in VANETs. Handover, which has been studied in cellular networks, occurs in the area between two neighbors BSs known as overlapping area [24]. This area resulted from overlap in the transmission range of both BSs and the signal in this area is weak because of interference in the frequencies of neighbor BSs, as shown in Fig. 1. Interference occurs between two neighbor BSs in the mobile cellular network, as proposed in [25]. Based on a literature review, no previous work has focused on handovers that occur in the overlapping area in the clustering of VANETs. Handover problem in clustering of VANETs is a more serious problem than in mobile devices because vehicles have ten times higher speed than mobile devices according to [10]. Vehicles in the overlapping area received two frequencies, one frequency from each BS, therefore vehicles are confused about sending its information to which base-station, while at the same time, the signal in this area has become weaker due to interference frequencies [25]. Three reasons why the handover problem occurs more frequently in VANETs is because vehicles are moving in high mobility, the vehicles are capable to move quickly from one BS to another, and vehicles require link established when moving, which cause increased handover in the network. According to Malaysian local cellular networks, the transmission range of $\mathrm{BS}$ in the urban city is limited so as to reduce the effect on people's health, and this has resulted in a high number of BSs, which is another reason to increase handover in the city. The use of clustering in this research has led to handover becoming a serious problem because communication with BS is done by cluster-head. In addition, the information that is sent to BSs consists of both $\mathrm{CH}$ and its $\mathrm{CMs}$ information, which may cause loss of cluster information when $\mathrm{CHs}$ is in the overlapping area. This is another reason that motivates us to solve this problem. Fig. 2 shows the handover problem in the overlapping area of clustering in VANETs.

From Fig. 2, $\mathrm{CH} 2$ is in the overlapping area, therefore it received two weak signals from two neighbor base-stations. Thus, during the handover with $\mathrm{CH} 2$, information of cluster is lost. The proposed method aimed to solve the above problem by using intelligent cluster-head ( $\mathrm{ICH}$ ) as discussed in the next section.

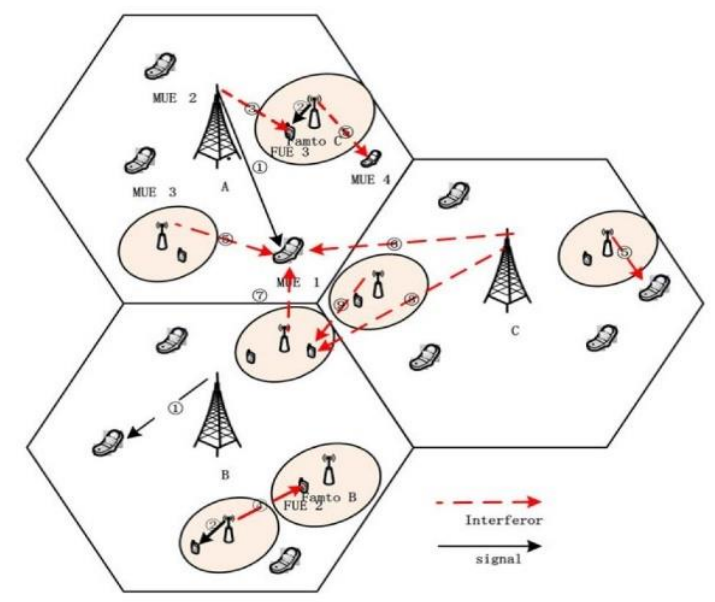

Fig. 1. Interference between Two BSs in the Mobile Network [25].

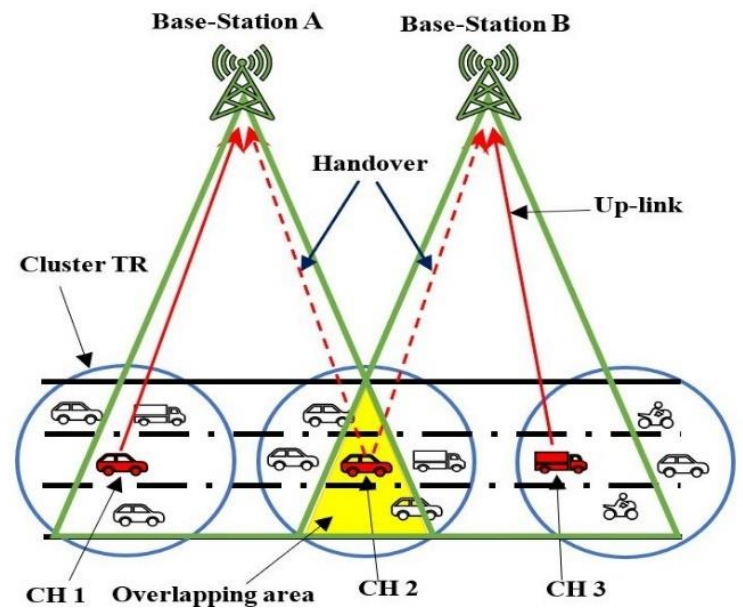

Fig. 2. Handover Problem in the Overlapping Area of Clustering in VANETs.

\section{RELATED WORKS}

This part introduced previous works that used clustering with cellular networks (heterogeneous networks) in VANETs. The main challenge in this area is high vehicle mobility that results in a dynamic topology change. This has raised several issues, which are the stability of the cluster, overhead, delay, and disconnect problem. In previous work, [11] proposed centralized clustering-based hybrid vehicular networking architecture (CC-HVNA) that combines both IEEE 802.11p and LTE in VANETs to enhance data dissemination by creating roadside units (RSUs) or BS to elect and form a cluster. This method resulted in improved delay and packet delivery ratio (PDR). The authors in [12] proposed hierarchical cluster-based location service in city environments (HCBLS) to reduce overhead and increase cluster stability. Overhead is reduced by reducing the update location costs. Stability is enhanced by the selection of vehicle in the centric of neighbor's vehicles, known as $\mathrm{CH}$. The outcome of this method is reduced location updates and increased cluster lifetime. In previous work, [13] proposed a novel multi-hop moving zone (MMZ) clustering scheme $\mathrm{CH}$ selection based on average relative speed, relative distance, and link life. The result of this method is increased $\mathrm{CH}$ lifetime and reduced delay. Moreover, authors in [14] proposed a new Vehicular Cloud (VC) model to enhance data dissemination by using LTE with IEEE802.11p, leading to increased PDR and reduced delay. Other authors in [15] proposed intelligent naïve Bayesian probabilistic estimation practice for traffic flow to form a stable clustering in VANET (ANTSC) algorithm to increase cluster stability by selected cluster head from the lane that has the heaviest traffic flow. In addition, authors in [16] proposed a novel destination and interest-aware clustering (DIAC) mechanism to reduce link failures between vehicles and LTE network based on the vehicle having the highest linkquality becoming the $\mathrm{CH}$. The authors in [17] proposed a hybrid vehicular multi-hop algorithm for stable clustering (VMaSC) with LTE and IEEE802.11p multi-hop clustering. LTE was used to increase PDR and reduce delay, while VMaSC was used to form a stable cluster by selecting $\mathrm{CH}$ based on the relative speed of vehicles in the same transmission range (TR). The combination of VMaSC with 
LET in a hybrid network, known as (VMaSC -LTE), was intended to reduce load at BS by reducing the number of clusters in the merge mechanism. However, while reduction in the number of clusters will reduce load at BS, the handover problem remains in the overlapping area. Other previous works focused on the use of gateway (GW) to reduce the load at BSs by a reduced number of $\mathrm{CHs}$ in the network. The $\mathrm{GW}$ is a normal node has two types based on the location of GW. The first type is the GW that is positioned between more than one $\mathrm{CHs}$. This type of $\mathrm{GW}$ is used to send the $\mathrm{CH}$ information to other $\mathrm{CHs}$ that are within its transmission range with the aim to make each $\mathrm{CH}$ know about the neighboring CHs [18]-[20]. However, these $\mathrm{CHs}$ are still connected to the BSs even though $\mathrm{GW}$ is available. The second type of $\mathrm{GW}$ is the $\mathrm{GW}$ has a location at the beginning and end of the transmission range of $\mathrm{CH}$. This type is not used to exchange information between $\mathrm{CHs}$ but is responsible for inter-cluster communication and used to inform its $\mathrm{CH}$ about new neighbor $\mathrm{CH}$ for merge mechanism [21]. The main goal of both types of GW is to achieve merge mechanism to merge several $\mathrm{CHs}$ in one $\mathrm{CH}$ to decrease the number of $\mathrm{CH}$ in the network according to a specific condition. The disadvantage of GW is that this rebroadcast caused flooding in the network. However, most researches used GW in multi-hop to increase cluster scalability and reduce the number of $\mathrm{CH}$ in the network. There is no procedure to select GW based only on the location of the node, therefore more than one GWs between two $\mathrm{CHs}$ caused more flooding and only one GW can do the same work of all the GWs. The concept of relay node (RN) is that a normal node is used to rebroadcast $\mathrm{CH}$ message to reach all $\mathrm{CMs}$ according to [22], [23]. The RN also caused flooding in the network. None of the previous related works have focused on handover problem that occurs in the overlapping area during using clustering in VANETs, therefore this study has proposed a new method call Intelligent Cluster-Head (ICH) to solve this problem. The concept of ICH is completely different from $\mathrm{GW}$ and RN. Table I shows the difference between GW, RN, and $\mathrm{ICH}$. Summary of related works is presented in Table II.

\section{PROPOSED METHOD}

This section discusses the proposed new method to solve the handover problem that occurs after cluster formation mentioned in the previous section. The aim of this new method, known as Intelligent Cluster-Head (ICH), is to control the connection link between $\mathrm{CH}$ as discussed in section (C).

\section{A. Features of ICH are}

- ICH works as a controller on $\mathrm{CHs}$ and has the capability to move connection between $\mathrm{CH}$ and $\mathrm{BS}$ from one $\mathrm{CH}$ to another $\mathrm{CH}$, thereby reserving signal strength (RSS) of $\mathrm{CHs}$ to prevent handover in the overlapping area because the proposed method can control on $\mathrm{CHs}$ by changing connection from $\mathrm{CH}$ has weak signal to another $\mathrm{CH}$ has good signal from BS.

- ICH is not a broadcast beacon that CHs use to broadcast for CMs to reduce flooding in the networks. However, ICH checks RSS in the beacons and when RSS of one $\mathrm{CHs}$ becomes weak, the ICH sends a notification to this $\mathrm{CH}$ about moving the connection to another $\mathrm{CH}$ during
$\mathrm{ICH}$ to guarantee the information of vehicles in the $\mathrm{CH}$ that has weak RSS is not consumed by overhead.

- $\mathrm{ICH}$ reduced the number of up-links between $\mathrm{CH}$ and BS by allowing only one $\mathrm{CH}$ to communicate with $\mathrm{BS}$, and another $\mathrm{CH}$ sends its vehicle information during $\mathrm{ICH}$ to the $\mathrm{CH}$ that has a connection with BS. With this method, the number of up-link connections is reduced by half as compared to that available in all previous methods that used a heterogeneous network.

- ICH calculated dynamic threshold speed for both clusters according to simple Equation. ICH elected in accurate method. The details of this point are presented in the next sub-section.

\section{B. Elected $\mathrm{ICH}$}

Should note, in this study clusters formation and $\mathrm{CH}$ election based on same method used in VMaSC-1hop in [17]. This study focuses on electing ICH after clusters formation and $\mathrm{CH}$ elected. When a vehicle received two beacon messages from different $\mathrm{CHs}$, it does not change its state directly like $\mathrm{GW}$. This vehicle checks the direction of new $\mathrm{CH}$; if it is in the opposite direction, the vehicle drops the beacon and continues as $\mathrm{CM}$ in its original $\mathrm{CH}$. However, if a beacon message is in the same direction, there are two cases according to Fig. 3.

In the first case, if the original $\mathrm{CH}(\mathrm{OCH})$ is in front of new $\mathrm{CH}(\mathrm{NCH})$, the vehicle that received a new beacon from $\mathrm{NCH}$ first checks the $\mathrm{NCH}$ speed; if the speed is less than $\mathrm{OCH}$ speed, the vehicle drops beacon and continues as CM. However, if $\mathrm{NCH}$ has a higher speed than $\mathrm{OCH}$ speed, the vehicle calculates dynamic threshold speed (Dthr) from Equation (1) and (2), then calculates the speed difference between $\mathrm{NCH}$ and $\mathrm{OCH}$ according to Equation (3). Table III shows the symbols of this paper.

$$
\begin{aligned}
& D_{\text {thro }}=|O C H-V S| \\
& D_{\text {thrN }}=|O C H-V S| \\
& D C H s=N C H-O C H
\end{aligned}
$$

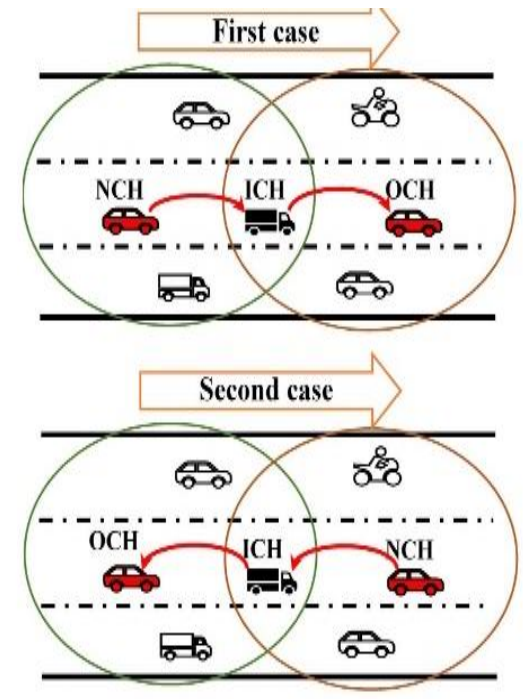

Fig. 3. Two Cases of $\mathrm{CH}$. 
TABLE. I. DIFFERENCE BETWEEN GW, RN AND ICH

\begin{tabular}{|c|c|c|c|}
\hline NO. & GW $[18]-[21]$ & RN [22], [23] & Proposed ICH \\
\hline 1 & Broadcast beacon and merge clusters & Only for broadcast beacon & $\begin{array}{l}\text { Controller by moving connection link between } \mathrm{CHs} \text { based } \\
\text { on RSS }\end{array}$ \\
\hline 2 & Increased flooding in the network & Increased flooding in the network & Avoid flooding beacon problem \\
\hline 3 & Reduced number of up-link if merge occur & Does not reduce the number of up-link & $\begin{array}{l}\text { Reduce the number of up-link without need for merge } \\
\text { mechanism }\end{array}$ \\
\hline 4 & Increased network scalability & Not did & Increased network scalability \\
\hline 5 & Elected based on location or $\mathrm{CH}$ elect GW & $\mathrm{CH}$ elect $\mathrm{RN}$ & $\begin{array}{l}\text { Elected according to a special procedure as shown in } \\
\text { section (B) }\end{array}$ \\
\hline 6 & Not used & Not used & $\begin{array}{l}\text { Used DDthr and DSthr to increase link lifetime between } \\
\text { CHs }\end{array}$ \\
\hline 7 & More than one in each $\mathrm{CH}$ or between $\mathrm{CHs}$ & More than one in each $\mathrm{CH}$ & Only one between $\mathrm{CHs}$ \\
\hline
\end{tabular}

TABLE. II. SUMMARY OF RELATED WORKS

\begin{tabular}{|c|c|c|c|c|c|}
\hline Ref. & Method & Problem & Domain & Outcome & $\begin{array}{l}\text { Solve handover } \\
\text { problem }\end{array}$ \\
\hline$[11]$ & CC-HVNA & - Data dissemination & Highway & $\begin{array}{l}\text { - Reduce Delay. } \\
\text { - Increase PDR. }\end{array}$ & NA \\
\hline [12] & HCBLS & $\begin{array}{l}\text { - Overhead. } \\
\text { - Stability }\end{array}$ & Urban & $\begin{array}{l}\text { - Increase cluster lifetime. } \\
\text { - Reduce location update. }\end{array}$ & NA \\
\hline [13] & MMZ & - Overhead & Highway & $\begin{array}{l}\text { - Increase CH lifetime. } \\
\text { - Reduce delay }\end{array}$ & NA \\
\hline$[14]$ & $\mathrm{VC}$ & - Data dissemination & NA & $\begin{array}{l}\text { - Increase PDR. } \\
\text { - Reduce delay. }\end{array}$ & NA \\
\hline$[16]$ & DIAC & - Link Failures & Urban & $\begin{array}{l}\text { - Increase } \mathrm{CH} \text { and } \mathrm{CM} \text { duration. } \\
\text { - Reduce overhead. } \\
\text { - Increase PDR. }\end{array}$ & NA \\
\hline [17] & VMaSC & $\begin{array}{l}\text { - Stability } \\
\text { - Overhead }\end{array}$ & Highway & $\begin{array}{l}\text { - Increase } \mathrm{CH} \text { duration. } \\
\text { - Reduce the number of cluster in the network. } \\
\text { - Increase PDR. } \\
\text { - Reduce delay. }\end{array}$ & NA \\
\hline
\end{tabular}

TABLE. III. LIST OF SYMBOLS

\begin{tabular}{|l|l|l|l|l|l|}
\hline NO. & Symbol & Description & NO. & Symbol & Description \\
\hline 1 & $\mathrm{OCH}$ & Original cluster-head & 13 & LLT $_{\mathrm{O}, \mathrm{I}}$ & Link lifetime between OCH and ICH \\
\hline 2 & $\mathrm{NCH}$ & New cluster-head & 14 & LLT $_{\mathrm{N}, \mathrm{I}}$ & Link lifetime between NCH and ICH \\
\hline 3 & $\mathrm{D}_{\mathrm{thrO}}$ & Dynamic threshold speed of $\mathrm{OCH}$ & 15 & $\Delta \mathrm{D}_{\mathrm{O}, \mathrm{N}}$ & The relative distance between OCH and NCH \\
\hline 4 & $\mathrm{D}_{\mathrm{thr}}$ & Dynamic threshold speed of NCH & 16 & $\mathrm{P}_{\mathrm{O}}$ & Position of OCH \\
\hline 5 & $\mathrm{VS}$ & Vehicle speed & 17 & $\mathrm{P}_{\mathrm{I}}$ & Position of ICH \\
\hline 6 & $\mathrm{DCHs}$ & Different CH speed & 18 & $\mathrm{P}_{\mathrm{N}}$ & Position of NCH \\
\hline 7 & $\Delta \mathrm{D}_{\mathrm{CH}, \mathrm{CM}}$ & The relative distance between CH and CM & 19 & $\Delta \mathrm{V}_{\mathrm{O}, \mathrm{N}}$ & The relative speed between OCH and NCH \\
\hline 8 & $\Delta \mathrm{V}_{\mathrm{CH}, \mathrm{CM}}$ & The relative speed between $\mathrm{CH}$ and CM & 20 & $\mathrm{~V}_{\mathrm{O}}$ & Speed of OCH \\
\hline 9 & $\mathrm{~V}_{\mathrm{CH}}$ & Speed of CH & 21 & $\mathrm{~V}_{\mathrm{I}}$ & Speed of ICH \\
\hline 10 & $\mathrm{~V}_{\mathrm{CM}}$ & Speed of CM & 22 & $\mathrm{~V}_{\mathrm{N}}$ & Speed of NCH \\
\hline 11 & $\mathrm{LLT}_{\mathrm{CH}, \mathrm{CM}}$ & Link lifetime between CH and its CM & 23 & $\mathrm{TR}_{\mathrm{O}}$ & Transmission range of OCH \\
\hline 12 & $\mathrm{LLT}_{\mathrm{O}, \mathrm{N}}$ & Link lifetime between $\mathrm{OCH}$ and NCH & 24 & $\mathrm{TR}_{\mathrm{I}}$ & Transmission range of ICH \\
\hline
\end{tabular}


When the difference in speed between $\mathrm{OCH}$ and $\mathrm{NCH}$ is less than dynamic threshold speed according to Equation (4), the vehicle sends message to $\mathrm{OCH}$ that contains all previous details. The $\mathrm{OCH}$ then checks how many vehicles have received beacon message from $\mathrm{NCH}$, then the Competing vehicles be made $\mathrm{ICH}(\mathrm{CICH})$; if there is only one vehicle, the $\mathrm{CH}$ sends a confirmation message to the vehicle and the vehicle becomes ICH. If there is more than one vehicle proposed to be $\mathrm{ICH}$, the $\mathrm{OCH}$ calculates the different dynamic threshold for each vehicle according to Equation (5). Vehicles with low different dynamic threshold speed, distance near to the half of transmission range (TR) become ICH. The remaining $\mathrm{CICH}$ vehicles are arranged in the table ranged from low different dynamic threshold speed to high, with the benefit that when ICH loses connection in any way, the vehicle in the second row of the table directly becomes ICH in order to not repeat the process again for more flexibility in the network.

DCHs $\leq D_{\text {thro }}$

$I C H=\left|D_{\text {thro }}-D_{\text {thrN }}\right|$
In the second case, the vehicle's original $\mathrm{CH}(\mathrm{OCH})$ backs off new $\mathrm{CH}(\mathrm{NCH})$. The vehicle that received a new beacon from $\mathrm{NCH}$ first checks $\mathrm{NCH}$ speed, and if this speed is greater than $\mathrm{OCH}$ speed, the vehicle drops the beacon and continues as CM. However, if $\mathrm{NCH}$ has a lower speed than $\mathrm{OCH}$ speed, the vehicle calculates dynamic threshold speed (Dthr) from Equation (1) and (2), then calculates different speed between $\mathrm{NCH}$ and $\mathrm{OCH}$ according to Equation (6). The same step above is used for Equation (4) and (5).

$\mathrm{DCHS}=\mathrm{OCH}-\mathrm{NCH}$

In the above cases, in this study, assume the vehicle that received a new beacon from another $\mathrm{CH}$ belongs to the $\mathrm{OCH}$. However, if vehicles belong to $\mathrm{NCH}$, the same above cases occur but the main difference is that validation will be with the value of $\mathrm{NCH}$. This means that in all cases, the validation is done with the value of $\mathrm{CH}$ that owned the $\mathrm{ICH}$. Fig. 4 shows the flowchart for elected ICH in both cases.

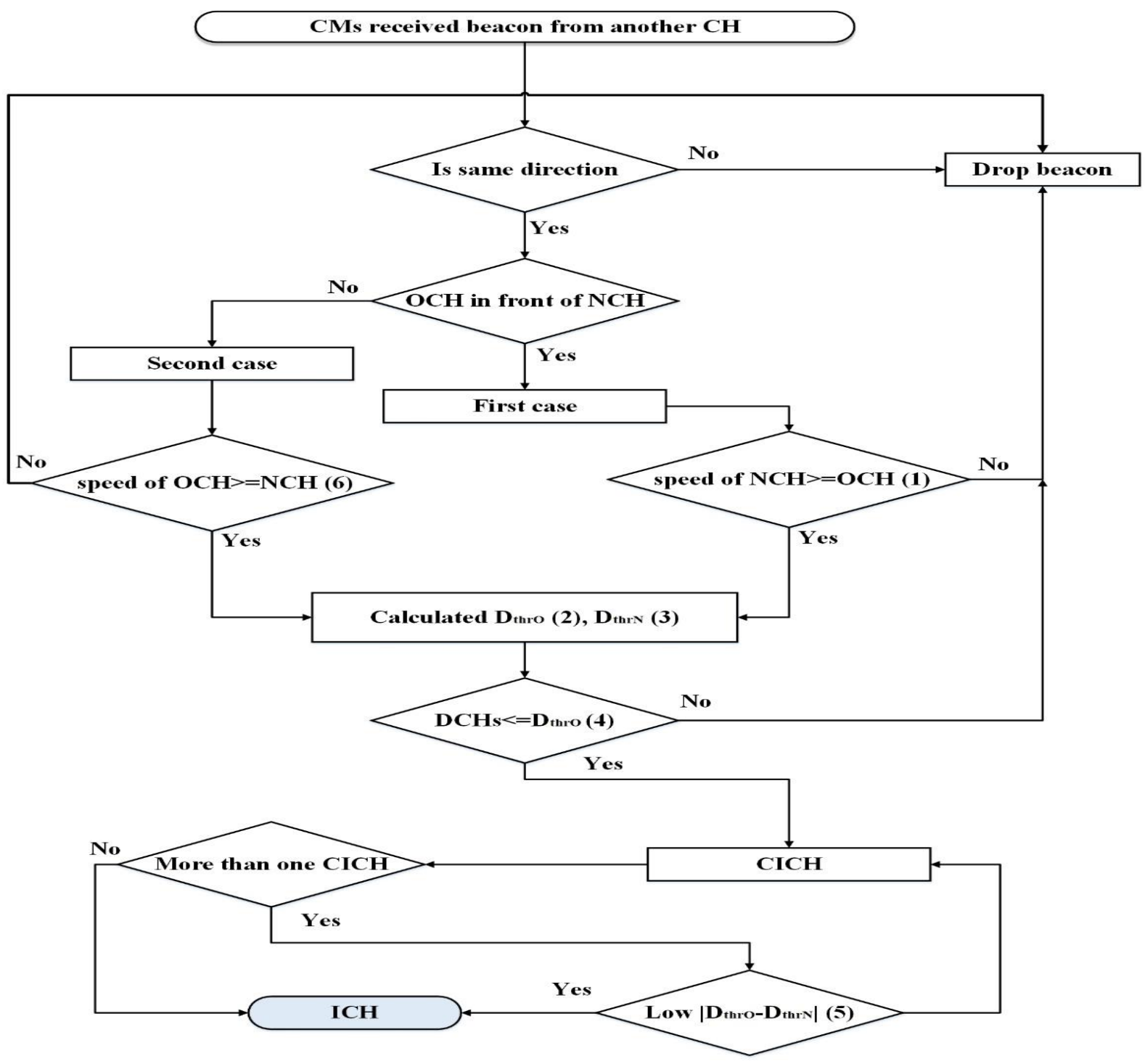

Fig. 4. Flowchart of ICH Election Process. 


\section{Solve Handover Problem by Applying ICH Method}

This section introduced how ICH manages neighbor clusters and changes up-link from one $\mathrm{CH}$ to another to solve the handover problem in the overlapping area. After $\mathrm{CH}$ sends a confirmation message to the proposed vehicle that has high qualification according to the previous section, the vehicle changes its state from $\mathrm{CM}$ to ICH then informs another cluster of this change. ICH begins to listen to both messages coming from $\mathrm{CHs}$, and this message has $\mathrm{CH}-\mathrm{ID}$, direction of $\mathrm{CH}$, speed of $\mathrm{CH}$, number of $\mathrm{CMs}$ in the $\mathrm{CH}$, different relative speed between $\mathrm{CH}$ and each $\mathrm{CM}$, different relative distance between $\mathrm{CH}$ and its $\mathrm{CMs}$, location of all clusters (location of each $\mathrm{CM}$ in addition to location of $\mathrm{CH}$ itself) and $\mathrm{RSS}$ of $\mathrm{CH}$. The $\mathrm{ICH}$ compares the RSS of both $\mathrm{CHs}$, and the $\mathrm{CH}$ having the highest RSS sends vehicle information for both clusters to the BS, while the $\mathrm{CH}$ that has low RSS sends its cluster information directly by using IEEE802.11p standard to ICH. The ICH sends this information to $\mathrm{CH}$ having the highest RSS. During this process, the $\mathrm{CH}$ that is connected in the BS remains connected in the BS until it has received two RSS from two BSs (this means the $\mathrm{CH}$ has arrived at the overlapping area). The $\mathrm{CH}$ then sends a weak signal message to $\mathrm{ICH}$ to change uplink connection with the $\mathrm{BS}$ to another $\mathrm{CH}$ in order not to lose vehicles' information. When $\mathrm{ICH}$ received a weak signal from $\mathrm{CH}$, the $\mathrm{ICH}$ sends a message to another $\mathrm{CH}$ to establish a link with the BS. The ICH sends vehicle information of $\mathrm{CH}$ having two signals to another cluster, and then another cluster begins to send information to the $\mathrm{BS}$ while $\mathrm{CH}$ that received two signals disconnects the uplink with BS. Fig. 5 shows how ICH works.

As shown in Fig. 5, CH 2 received two signals because it is in the overlapping area. Thus, $\mathrm{CH} 2$ sends a weak signal message to $\mathrm{ICH}$, which then sends a message to $\mathrm{CH} 1$ to establish a link with the BS to send vehicle information. After the link has been established, the ICH sends $\mathrm{CH} 2$ information to $\mathrm{CH} 1$, which is then sent to $\mathrm{BS}$. By using $\mathrm{ICH}$, the handover problem that occurs in the overlapping area is solved. Also, the use of $\mathrm{ICH}$ reduced the number of up-link connections with the $\mathrm{BS}$, thereby increasing the cluster stability in the network. Fig. 6 shows the flowchart of vehicles that work as ICH.

Based on previous work [26], the LLT between $\mathrm{CH}$ and $\mathrm{CM}$ is collected from the following equation.

$\Delta D_{C H, C M}=\left|P_{C H}-P_{C M}\right|$

$\Delta V_{C H, C M}=\left|V_{C H}-V_{C M}\right|$

Collected LLT between $\mathrm{CH}$ and $\mathrm{CM}$ has two cases according to the following:

1) First case: When CM is in back of CH, LLT is collected according to Equation (9) in [26]

$L L T_{C H, C M}=\frac{\Delta V_{C H, C M} * \Delta D_{C H, C M}+\Delta V_{C H, C M} * T R_{C H}}{\left(\Delta V_{C H, C M}\right)^{2}}$

2) Second case: When CM is in front of CH, LLT is collected according to Equation (10) in [26].

$L L T_{C H, C M}=\frac{-\Delta V_{C H, C M} * \Delta D_{C H, C M}+\Delta V_{C H, C M} * T R_{C H}}{\left(\Delta V_{C H, C M}\right)^{2}}$

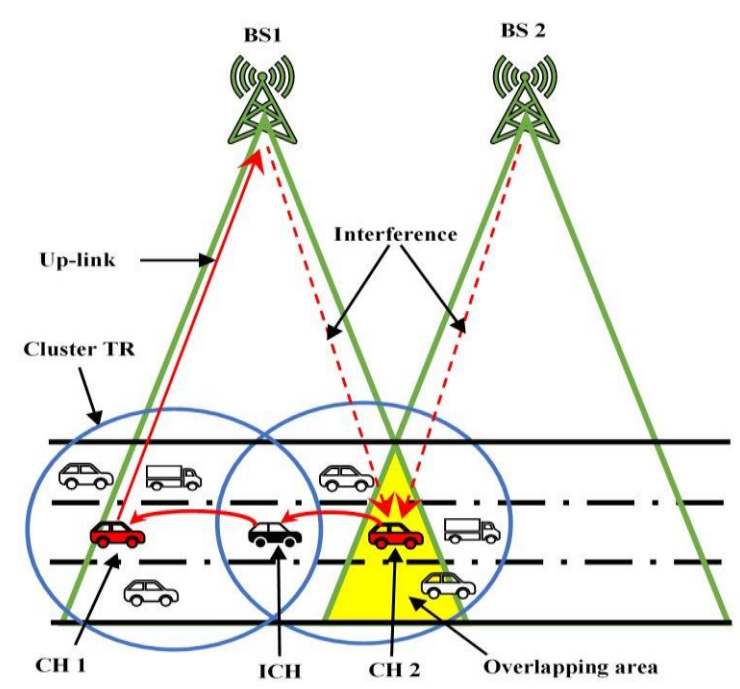

Fig. 5. Work of Intelligent Cluster-Head (ICH).

In this method, LLT is collected between two $\mathrm{CHs}$ during ICH. Also, there are two cases to collect LLT according to Fig. 3. The total LLT between $\mathrm{OCH}$ and $\mathrm{NCH}$ in this study was calculated according to Equation (11), while the different distance and different velocity were calculated according to Equation (12) and (13), respectively.

$$
\begin{aligned}
& L L T_{O, N}=L L T_{O, I}+L L T_{N, I} \\
& \Delta D_{O, N}=\left|P_{O}-P_{I}\right|+\left|P_{N}-P_{I}\right| \\
& \Delta V_{O, N}=\left|V_{O}-V_{I}\right|+\left|V_{N}-V_{I}\right|
\end{aligned}
$$

3) The first case according to Fig. 3: each case has two LLTs, one between $\mathrm{OCH}$ and ICH, while another LLT is between $\mathrm{NCH}$ and $\mathrm{ICH}$. This means this method that the $\mathrm{ICH}$ must be in front of one $\mathrm{CH}$ and behind another $\mathrm{CH}$. In Fig. 3, in this study, assume ICH belongs to $\mathrm{OCH}$, only in both cases the purpose is to validate with the value of $\mathrm{OCH}$ only. The same scenario applies when ICH belongs to $\mathrm{NCH}$ exactly but validated with the value of $\mathrm{NCH}$. According to Fig. 3, the $\mathrm{ICH}$ is behind $\mathrm{OCH}$; therefore the LLT is collected according to Equation (14). In the same case where the $\mathrm{ICH}$ is in front of $\mathrm{NCH}$, the LLT is collected according to Equation (15).

When ICH is behind OCH, LLT is collected according to Equations (8), (9) and (10).

$L L T_{O, I}=\frac{\Delta V_{O, I} * \Delta D_{O, I}+\Delta V_{O, I} * T R_{O}}{\left(\Delta V_{O, I}\right)^{2}}$
$L L T_{N, I}=\frac{-\Delta V_{N, I} * \Delta D_{N, I}+\Delta V_{N, I} * T R_{I}}{\left(\Delta V_{N, I}\right)^{2}}$

To get the total LLT between two $\mathrm{CHs}(\mathrm{OCH}$ and $\mathrm{NCH})$, substitute Equations (14) and (15) in Equation (11).

$L L T_{O, N}=\frac{\Delta V_{O, I} * \Delta D_{O, I}+\Delta V_{O, I} * T R_{I}}{\left(\Delta V_{O, I}\right)^{2}}+\frac{-\Delta V_{N, I} * \Delta D_{N, I}+\Delta V_{N, I} * T R_{I}}{\left(\Delta V_{N, I}\right)^{2}}$

Simplify equation (16) to get the best equation to collect LLT between two CHs in Equation (17). 


$$
\begin{gathered}
L L T_{O, N}=\frac{\Delta V_{O, I} *\left(\Delta D_{O, I}+T R_{I}\right)}{\left(\Delta V_{O, I}\right)^{2}}+\frac{\Delta V_{N, I} *\left(-\Delta D_{N, I}+T R_{I}\right)}{\left(\Delta V_{N, I}\right)^{2}} \\
=\frac{\Delta D_{O, I}+T R_{I}}{\left(\Delta V_{O, I}\right)}+\frac{-\Delta D_{N, I}+T R_{I}}{\left(\Delta V_{N, I}\right)} \\
=\frac{\left(\Delta V_{N, I}\right) *\left(\Delta D_{O, I}+T R_{I}\right)+\left(\Delta V_{O, I}\right) *\left(-\Delta D_{N, I}+T R_{I}\right)}{\left(\Delta V_{O, I}\right) *\left(\Delta V_{N, I}\right)} \\
=\frac{\Delta V_{N, I} * \Delta D_{O, I}+\Delta V_{N, I} * T R_{I}-\Delta D_{N, I} * \Delta V_{O, I}+\Delta V_{O, I} * T R_{I}}{\left(\Delta V_{O, I}\right) *\left(\Delta V_{N, I}\right)}
\end{gathered}
$$

4) The second case according to Fig. 3: this case is different than other cases in that the signals are based on the location of $\mathrm{ICH}$ according to $\mathrm{OCH}$ and $\mathrm{NCH}$ as shown in Equation (18).

$$
L L T_{O, N}=\frac{-\Delta V_{O, I} * \Delta D_{O, I}+\Delta V_{O, I} * T R_{O}}{\left(\Delta V_{O, I}\right)^{2}}+\frac{\Delta V_{N, I} * \Delta D_{N, I}+\Delta V_{N, I} * T R_{I}}{\left(\Delta V_{N, I}\right)^{2}}
$$

\section{Theoretical Analysis and a Numerical Example}

Based on the real-data collected from local cellular networks in Batu Pahat, Johor, Malaysia, the transmission range of LTE-base-station is between 300 to 400 meters. Therefore, there are many BSs on the road, which leads to a lot of overlapping areas that resulted in more handovers as vehicles move on the road. Handover caused serious problems, especially in clustering, because each cluster represents the $\mathrm{CH}$ of each vehicle, and passing $\mathrm{CH}$ in the overlapping area caused loss of all CMs information resulted from handover between two BSs. [17] proposed VMaSC-LTE that is closest to the idea of this study, where the load on the BS is reduced due to a number of clusters in the network. However, reduction in the number of clusters will reduce load at BS but not solve handover problem because the remaining clusters still have a handover. Also, there has been no focus on the overlapping area. In this paper, we proposed ICH to solve handover problems by positioning the ICH at the end of clusterstransmission range. Thus, the front $\mathrm{CH}$ that received two signals from two different BSs is directed to stop sending vehicle information to the $\mathrm{BS}$ in order to not lose its cluster information and sends this information to $\mathrm{ICH}$ and then to another $\mathrm{CH}$ that is not in the overlapping area. Also, ICH reduced uplink by half because every two clusters that have ICH use only one uplink to connect both clusters in the BS. This is because of the use of IEEE802.11p protocol to connect inter and intra cluster and this protocol has data rate $27 \mathrm{Mbps}$ according to [27]. This study focuses on broadcast safety message that has a size between (512 and 1000) byte according to [10], [14], [17], [28], therefore the link between $\mathrm{CH}$ and BS can send both $\mathrm{CHs}$ information during IEEE502.11p. For example, if each $\mathrm{CH}$ has a maximum number of $\mathrm{CMs}$, this means $20 \mathrm{CMs}$ according to [29], therefore the total number of both clusters within the $\mathrm{CHs}$ becomes 42 vehicles. According to above, each vehicle sends safety message 1000 bytes in size, therefore the total size of safety message for both clusters become $(42 \times 1000=42,000$ bytes. Divided by 1000 to convert to $\mathrm{KB}$, the size becomes $42 \mathrm{Kbps}$ and the link can send up to $27 \mathrm{Mbps})$. Also, a reduced number of up-link results in reduced overhead cost and increased cluster stability in the network. Since VMaSC-LTE is used in highway scenario, in order to evaluate this method with VMaSC-LTE, the idea of this method was applied in this paper, scenario in the numerical example.

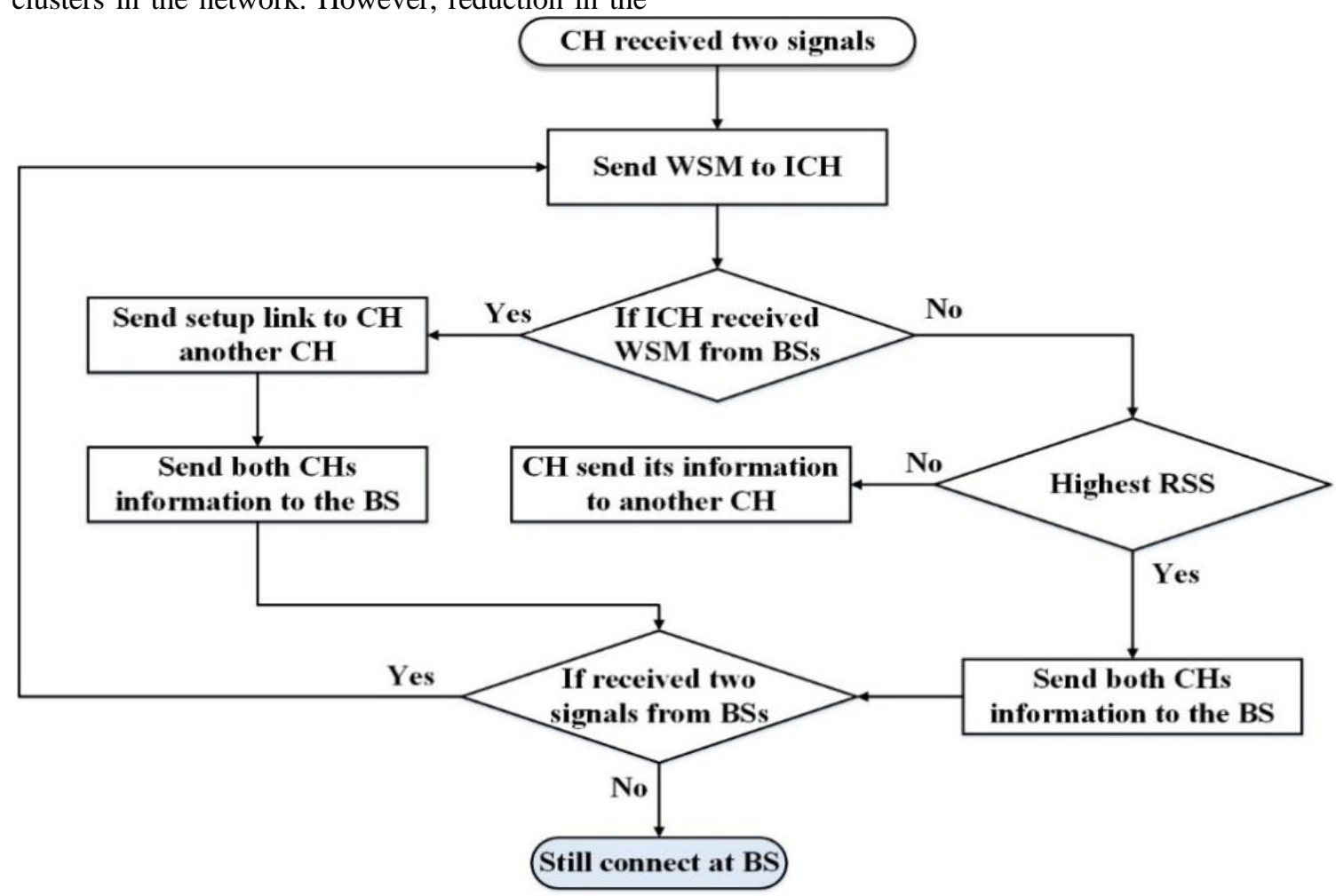

Fig. 6. Flowchart of Vehicles that Work as ICH. 
If assumed length of road to be $10 \mathrm{~km}$ in urban Batu Pahat, Johor, Malaysia, and number of vehicles $(\mathrm{N}-\mathrm{V})$ to be 100 according to real-data of local cellular network in Batu Pahat, the TR of BS $300 \mathrm{~m}$, the number of BSs (N-BS) is closer to 33 $\mathrm{BS}$ according to the real-data collected from local cellular networks. This resulted in 32 overlapping area (OA), the number of CMs (N-CMs) in each cluster is 20; therefore the total number of CHs (N-CHS) in this road is 5. These clusters in VMaSC-LTE have handover (HO) in each overlapping area. Therefore, a number of handovers in VMaSC-LTE are $32 \times 5 \times 20=3200$ handovers for total cluster according to the number of CMs in each cluster over a $10 \mathrm{~km}$ road from 100 vehicles. However, in this method, no handover occurs in the clusters having $\mathrm{ICH}$, but the handover occurs if the cluster does not have ICH. However, most of the clusters in proposed method have ICH because of high vehicle density in the urban area, especially in Batu Pahat city. From the above example and because the $\mathrm{ICH}$ connects only two $\mathrm{CHs}$, in the above example one of five $\mathrm{CH}$ has handover in the overlapping area. Therefore, the number of packets lost in this cluster is calculated according to $32 \times 1 \times 20=640$ handover in the network on the same road.

As shown in Fig. 7(A), there is no handover in the overlapping area because ICH changed the up-link when $\mathrm{CH} 2$ received two signals from $\mathrm{BS} 1$ and $\mathrm{BS} 2$. However, in Fig. 7(B), the $\mathrm{CH} 2$ has no other choice than to send its cluster information, therefore the probability of handover occurrence is high. The number of handovers increases as road length and number of vehicles on the road increases. Table IV shows the result of a numerical example.
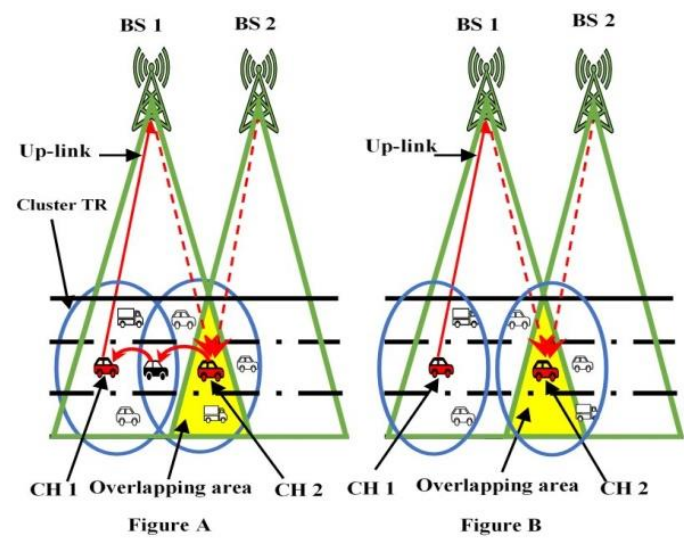

Fig. 7. Illustration of the difference between ICH and VMaSC-LTE Methods.

TABLE. IV. RESULT OF A NUMERICAL EXAMPLE.

\begin{tabular}{|l|l|l|l|l|l|l|}
\hline Method & Road & N-V & N-CH & N-BS & OA & HO \\
\hline VMaSC-LTE [17] & $10 \mathrm{~km}$ & 100 & 5 & 33 & 32 & 3200 \\
\hline ICH & $10 \mathrm{~km}$ & 100 & 5 & 33 & 32 & 640 \\
\hline
\end{tabular}

\section{Simulation Modeling}

In this section, evaluated and validated ICH method with VMaSC-1hop method [17]. The evaluation was done by applying the concept of VMaSC-1hop on the scenario. VMaSC-1hop method has been selected for evaluation because it is close to the proposed idea. The VMaSC-1hop used GW to merge clusters to reduce load at BSs, which consist of packet loss, packet delivery ratio, disconnect problem and number of uplink connection. Unfortunately, this method did not discuss the handover problem that occurs in the overlapping area. Therefore, the proposed method reduces the load at BSs in the overlapping area. Both methods are applied by using MATLAB-a 2018 in the simulation. The simulation parameters used in this study are shown in Table V.

\section{A. Performance Metrics}

- Packet loss: This is defined as the number of $\mathrm{CHs}$ that failed in sending the cluster information to the BSs. In this study, the packet loss is measured in the overlapping area (cell edges) only. Also, the information related to $\mathrm{CHs}$ loss in this area consists of information of $\mathrm{CH}$ and its CMs. The packet loss increased when the number of clusters increased and the number of CMs in each $\mathrm{CH}$ increased.

- Packet delivery ratio (PDR): This is defined as the number of $\mathrm{CHs}$ that successfully sent its cluster information to the BS during cell edge. The PDR increased when the number of $\mathrm{CH}$ that successfully sent its cluster information in the cell edge increased and the number of $\mathrm{CMs}$ in each $\mathrm{CH}$ increased.

- A number of disconnections: It is the number of $\mathrm{CH}$ that lost connection with BS during cell edges from the total number of $\mathrm{CH}$ that successfully connects with the BS in this area.

- Network efficiency: The average ratio of a number of packet loss to the total number of PDR in the cells edge. Increased percentage of packet loss during cells edge result in reduced network efficiency.

\section{B. Performance Comparison between ICH Method and VMaSC-1hop Method}

Table VI shows the ICH method has less packet loss than VMaSC-1hop method in cells edge because of the ICH transfer communication up link from $\mathrm{CH}$ that received two signals from different BSs to another $\mathrm{CH}$. By this process, the number of packet loss is reduced. The VMaSC method used GW to make the connection between neighbors' $\mathrm{CHs}$ for merge mechanism without any permission to move up link connection because it is a normal node; therefore, the packet loss occurs in the cells edge. From the table, the ICH method also has packet loss, but much less than VMaSC method. The packet loss in the proposed method results from $\mathrm{CH}$ that had no neighbor $\mathrm{CH}$. Therefore, in this case, the packet loss occurred in $\mathrm{ICH}$ method. The average percentage of packet loss at a cell edge in the ICH method and VMaSC-1hop method is $0.8 \%$ and $84 \%$, respectively.

Fig. 8 shows the ICH method has higher packet delivery ratio than the previous method, and the reason is that proposed method can deliver packets even in cell edge by changing uplink from $\mathrm{CH}$ that has weak signal or confused single to $\mathrm{CH}$ that has good signal or only one signal. Thus, the percentage of PDR in proposed method is greater than the previous method. The average percentage of PDR at the cell edge in ICH method and VMaSC-1hop method is $99 \%$ and $15 \%$, respectively. 
TABLE. V. SIMULATION PARAMETERS

\begin{tabular}{|l|l|}
\hline Parameters & Value \\
\hline Simulation time & $300 \mathrm{~s}$ in each run \\
\hline MAC protocol & IEEE $802.11 \mathrm{p}$ \\
\hline Transmission range & $300 \mathrm{~m}$ \\
\hline Number of vehicles & $100,200,300,400,575$ \\
\hline Road length & $17.8 \mathrm{~km}$ \\
\hline Number of lanes in the road & 3 \\
\hline Length of car & $3 \mathrm{~m}$ \\
\hline Maximum lane speed & $10-100 \mathrm{~km} / \mathrm{h}$ \\
\hline Number of hops & One hope \\
\hline maximum number of CMs in each CH & 20 \\
\hline Number of iterations & 100 \\
\hline Number of runs & 10 \\
\hline
\end{tabular}

TABLE. VI. Number of PACKet Loss In Both Methods In the Cells EDGE

\begin{tabular}{|l|l|l|}
\hline Number of Vehicles & Proposed & VMaSC-1hop [17] \\
\hline 100 Vehicles & 181 & 6920 \\
\hline 200 Vehicles & 167 & 16422 \\
\hline 300 Vehicles & 151 & 25314 \\
\hline 400 Vehicles & 190 & 34466 \\
\hline 575 Vehicles & 149 & 50467 \\
\hline $\begin{array}{l}\text { The average number of packet loss } \\
\text { in the cell edge }\end{array}$ & 167.60 & 26717.8 \\
\hline
\end{tabular}

Fig. 9 shows the number of disconnect in the cells edge between ICH method and VMaSC-1hop method. The ICH method resulted in fewer disconnect than the previous method because the ICH allows $\mathrm{CH}$ to connect in the BS, even the $\mathrm{CH}$ in the cell edge, while the previous method during cell edge has disconnect because the $\mathrm{CH}$ received two signals from different $\mathrm{BS}$ and $\mathrm{GW}$ cannot transfer uplink connection to neighbor $\mathrm{CH}$.

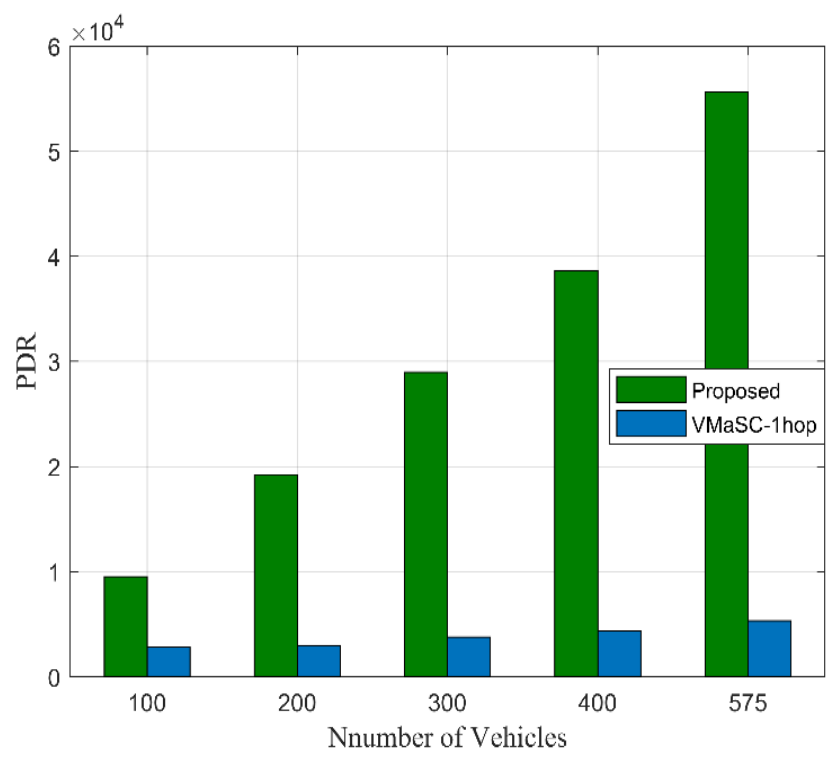

Fig. 8. PDR in the Cells Edge.

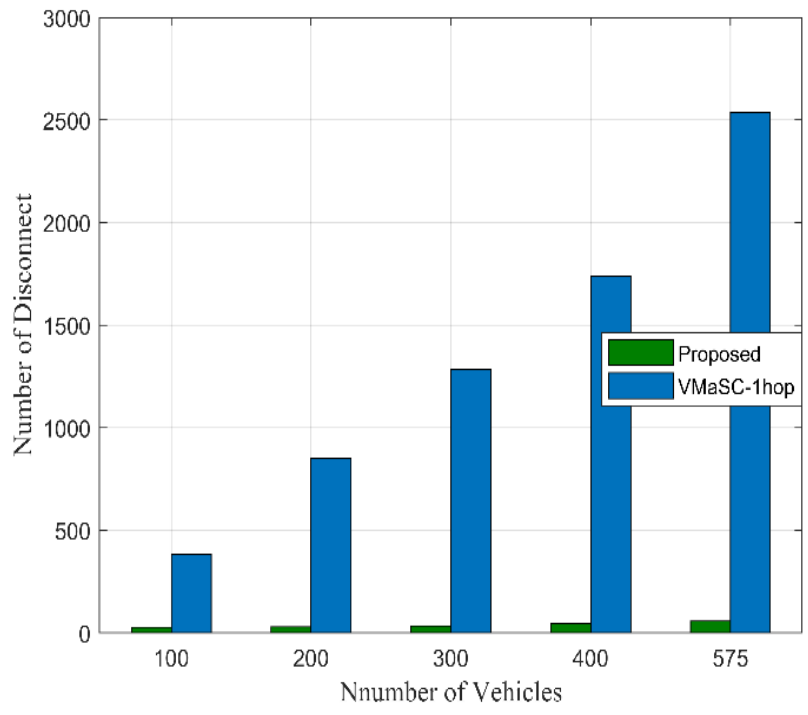

Fig. 9. Disconnect Problem During Cells Edge.

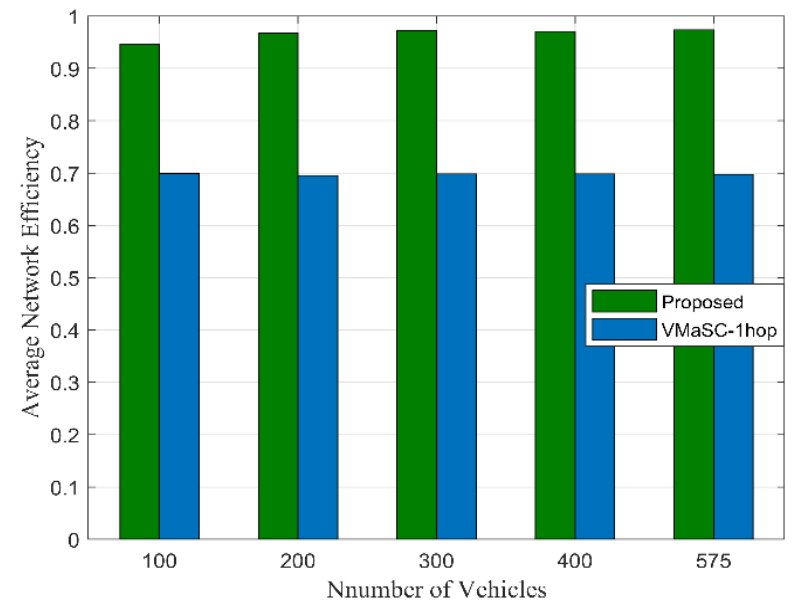

Fig. 10. Network Efficiency in the Cells Edge.

Fig. 10 shows the network efficiency of ICH and VMaSC1hop method. From the figure, the ICH method results in a higher percentage of network efficiency than VMaSC-1hop method because ICH method has less percentage of packet loss, less percentage of disconnect problem and a higher percentage of PDR than VMaSC-1hop method. Therefore, the network efficiency in ICH method is higher than in VMaSC1 hop method. The average percentage of network efficiency in the ICH method and VMaSC-1hop method is $96 \%$ and $70 \%$, respectively.

\section{CONCLUSION}

This paper has proposed a novel method known as Intelligent Cluster-Head to solve the handover problem that occurs in the overlapping area (cell edge) when the cluster is passed from one BS to another neighbor BS. ICH is a controller vehicle that controls a neighbor's $\mathrm{CHs}$ and specifies the cluster having the higher RSS to send vehicle information of both clusters to BS. Also, ICH has the ability to change uplink from one cluster to another to solve handover problem of $\mathrm{CH}$ receiving two signals from different $\mathrm{BSs}$ in direct 
contact with $\mathrm{ICH}$, and the $\mathrm{ICH}$ then changes the uplink to another cluster. The evaluation was done by using MATLAB software during evaluating the ICH method with the concept of VMaSC-1hop and the result shows the ICH method and VMaSC-1hop have an average percentage of packet loss of $0.8 \%$ and $84 \%$, respectively. The percentage of PDR in $\mathrm{ICH}$ method and VMaSC-1hop is $99 \%$ and $15 \%$, respectively. The number of disconnect in ICH method is less than VMaSC-1hop method and the network efficiency in ICH method VMaSC1hop method is $96 \%$ and $70 \%$, respectively. In future work, will analyse and evaluate the effect of delay and overhead when using the proposed method. Also, we will apply this method on a highway scenario.

\section{REFERENCES}

[1] V. Vukadinovic, K. Bakowski, P. Marsch, I. D. Garcia, H. Xu, M. Sybis, P. Sroka, K. Wesolowski, D. Lister, and I. Thibault, "3GPP C-V2X and IEEE $802.11 \mathrm{p}$ for Vehicle-to-Vehicle communications in highway platooning scenarios," Ad Hoc Networks, vol. 74, pp. 17-29, 2018.

[2] G. Yan and D. B. Rawat, "Vehicle-to-vehicle connectivity analysis for vehicular ad-hoc networks," Ad Hoc Networks, vol. 58, pp. 25-35, 2017.

[3] E. Ndashimye, S. K. Ray, N. I. Sarkar, and J. A. Gutiérrez, "Vehicle-toinfrastructure communication over multi-tier heterogeneous networks: a survey," Comput. Networks, vol. 112, pp. 144-166, 2017.

[4] J. Hoeft Michałand Rak, "How to provide fair service for V2I communications in VANETs?," Ad Hoc Networks, vol. 37, pp. 283-294, 2016.

[5] Y. A. Shah, H. A. Habib, F. Aadil, M. F. Khan, M. Maqsood, and T. Nawaz, "CAMONET: Moth-flame optimization (MFO) based clustering algorithm for VANETs," IEEE Access, vol. 6, pp. 48611-48624, 2018.

[6] S. Vodopivec, J. Bešter, and A. Kos, "A survey on clustering algorithms for vehicular ad-hoc networks," in Telecommunications and Signal Processing (TSP), 2012 35th International Conference on, 2012, pp. 5256.

[7] S. M. AlMheiri and H. S. AlQamzi, "MANETs and VANETs clustering algorithms: A survey," in GCC Conference and Exhibition (GCCCE), 2015 IEEE 8th, 2015, pp. 1-6.

[8] M. Gerla and J. T.-C. Tsai, "Multicluster, mobile, multimedia radio network," Wirel. networks, vol. 1, no. 3, pp. 255-265, 1995.

[9] G. V Rossi, Z. Fan, W. H. Chin, and K. K. Leung, "Stable clustering for ad-hoc vehicle networking," in Wireless Communications and Networking Conference (WCNC), 2017 IEEE, 2017, pp. 1-6.

[10] A. Abuashour and M. Kadoch, "Performance improvement of clusterbased routing protocol in VANET," IEEE Access, vol. 5, pp. 1535415371, 2017.

[11] C. Shi, Y. Zhou, W. Li, H. Li, N. Lu, N. Cheng, and T. Yang, "A Centralized Clustering Based Hybrid Vehicular Networking Architecture for Safety Data Delivery," in GLOBECOM 2017-2017 IEEE Global Communications Conference, 2017, pp. 1-6.

[12] R. Aissaoui, A. Dhraief, A. Belghith, H. Menouar, H. Mathkour, F. Filali, and A. Abu-Dayya, "Hcbls: A hierarchical cluster-based location service in urban environment," Mob. Inf. Syst., vol. 2015, 2015.

[13] Z. Khan and P. Fan, "A multi-hop moving zone (MMZ) clustering scheme based on cellular-V2X," China Commun., vol. 15, no. 7, pp. 5566, 2018.
[14] S. A. Khawatreh and E. N. Al-Zubi, "Improved Hybrid Model in Vehicular Clouds based on Data Types (IHVCDT)," Int. J. Adv. Comput. Sci. Appl., vol. 8, no. 8, pp. 114-118, 2017.

[15] A. Mehmood, A. Khanan, A. H. H. M. Mohamed, S. Mahfooz, H. Song, and S. Abdullah, "ANTSC: An intelligent naive Bayesian probabilistic estimation practice for traffic flow to form stable clustering in VANET," IEEE Access, vol. 6, pp. 4452-4461, 2018.

[16] I. Ahmad, R. M. Noor, I. Ahmedy, S. A. A. Shah, I. Yaqoob, E. Ahmed, and M. Imran, "VANET LTE based heterogeneous vehicular clustering for driving assistance and route planning applications," Comput. Networks, vol. 145, pp. 128-140, 2018.

[17] S. Ucar, S. C. Ergen, and O. Ozkasap, "Multihop cluster based IEEE $802.11 \mathrm{p}$ and LTE hybrid architecture for VANET safety message dissemination," IEEE Trans. Veh. Technol., vol. 65, no. 4, pp. 26212636, 2016.

[18] R. S. Bali, N. Kumar, and J. J. P. C. Rodrigues, "An efficient energyaware predictive clustering approach for vehicular ad hoc networks," Int. J. Commun. Syst., vol. 30, no. 2, p. e2924, 2017.

[19] S.-S. Wang and Y.-S. Lin, "PassCAR: A passive clustering aided routing protocol for vehicular ad hoc networks," Comput. Commun., vol. 36, no. 2, pp. 170-179, 2013.

[20] M. Song and F. Cuckov, "A Mobility-Aware General-Purpose Vehicular Ad-Hoc Network Clustering Scheme.," J. Inf. Sci. Eng., vol. 26, no. 3, pp. 897-911, 2010.

[21] M. Ren, L. Khoukhi, H. Labiod, J. Zhang, and V. Vèque, “A mobilitybased scheme for dynamic clustering in vehicular ad-hoc networks (VANETs)," Veh. Commun., vol. 9, pp. 233-241, 2017.

[22] D. Al-Terri, H. Otrok, H. Barada, M. Al-Qutayri, R. M. Shubair, and Y. Al-Hammadi, "Qos-olsr protocol based on intelligent water drop for vehicular ad-hoc networks," in Wireless Communications and Mobile Computing Conference (IWCMC), 2015 International, 2015, pp. 13521357.

[23] E. Dror, C. Avin, and Z. Lotker, "Fast randomized algorithm for 2-hops clustering in vehicular ad-hoc networks," Ad Hoc Networks, vol. 11, no. 7, pp. 2002-2015, 2013.

[24] S. Sbit, M. B. Dadi, and B. C. Rhaimi, "Comparison of Inter Cell Interference Coordination Approaches," World Acad. Sci. Eng. Technol. Int. J. Electr. Comput. Energ. Electron. Commun. Eng., vol. 11, no. 7, pp. 865-870, 2017.

[25] Y. Li, C. Niu, F. Ye, and R. Q. Hu, "A universal frequency reuse scheme in LTE-A heterogeneous networks," Wirel. Commun. Mob. Comput., vol. 16, no. 17, pp. 2839-2851, 2016.

[26] Z. Y. Rawashdeh and S. M. Mahmud, "A novel algorithm to form stable clusters in vehicular ad hoc networks on highways," EURASIP J. Wirel. Commun. Netw., vol. 2012, no. 1, p. 15, 2012.

[27] D. Roy, M. Chatterjee, and E. Pasiliao, "Video quality assessment for inter-vehicular streaming with IEEE $802.11 \mathrm{p}$, LTE, and LTE Direct networks over fading channels," Comput. Commun., vol. 118, pp. 69-80, 2018.

[28] L. Rui, Y. Zhang, H. Huang, and X. Qiu, "A New Traffic Congestion Detection and Quantification Method Based on Comprehensive Fuzzy Assessment in VANET.," KSII Trans. Internet Inf. Syst., vol. 12, no. 1, 2018.

[29] S. Asoudeh, M. Mehrjoo, N.-M. Balouchzahi, and A. Bejarzahi, "Location service implementation in vehicular networks by nodes clustering in urban environments," Veh. Commun., vol. 9, pp. 109-114, 2017. 